\title{
Contribuiçóes da gameterapia para as habilidades cognitivas de um adolescente com paralisia cerebral ${ }^{1}$
}

\author{
Thiago da Silva Dias ${ }^{a}$ (D), Karoline Faro da Conceiçáo ${ }^{b}$ (D), Ana Irene Alves de Oliveira ${ }^{\mathrm{b}, c}$ (D), \\ Rafael Luiz Morais da Silva ${ }^{c, d}$ (i)
}

${ }^{a}$ Centro de Reabilitação Neurológica Matheus Álvares, Praia Grande, SP, Brasil.

${ }^{b}$ Unidade de Ensino e Assistência em Fisioterapia e Terapia Ocupacional - UEAFTO, Centro Especializado em Reabilitação Tipo II - CER II, Belém, PA, Brasil.

'Universidade do Estado do Pará - UEPA, Belém, PA, Brasil.

dUnidade de Ensino e Assistência em Fisioterapia e Terapia Ocupacional - UEAFTO, Universidade do Estado do Pará - UEPA, Belém, PA, Brasil.

\begin{abstract}
Resumo: Este estudo visa analisar as contribuições da gameterapia para as habilidades cognitivas de um adolescente com paralisia cerebral. Esta pesquisa é baseada em um método de estudo de caso, na qual foi utilizado o software Desenvolve ${ }^{\circledR}$ para comparar as habilidades cognitivas do sujeito antes e após 10 sessões de gameterapia utilizando cinco minijogos do jogo Big Brain Academy: Wii Degree. Observou-se progressão nos escores em 10 dentre as 19 habilidades cognitivas avaliadas. Houve progressão em cinco habilidades diretamente estimuladas pelos jogos utilizados. Com relação ao escore total, houve progressão de 73,69\% para 90,89\%. Desse modo, verificou-se ganhos nas habilidades cognitivas do sujeito após as sessões de gameterapia. Além disso, o participante se mostrou mais engajado e motivado para participar da terapia, bem como, conseguiu melhorar a jogabilidade no vídeo game, o qual faz parte de uma atividade recorrente no seu cotidiano e significativa para ele.
\end{abstract}

Palavras-chave: Disfunção Cognitiva, Jogos de Vídeo, Reabilitação, Tecnologia.

\section{Contributions of game therapy for cognitive skills of an adolescent with cerebral palsy}

\begin{abstract}
This study aims to analyze the contributions of game therapy for cognitive skills of an adolescent with cerebral palsy. This research is based on a case study method, in which the software Desenvolve ${ }^{\circledR}$ was used to compare the participant's cognitive skills before and after 10 game therapy sessions using five minigames from the videogame Big Brain Academy: Wii Degree. We found a percentage increase in the participant's scores regarding 10 out of 19 cognitive skills that had been assessed. Five of these skills had been directly stimulated through the intervention. Regarding the total score, we found a percentage increase from $73.69 \%$ to $90.89 \%$. Therefore, the study subject showed improvement in cognitive skills after the game therapy sessions. In addition, the subject became more engaged and motivated during the treatment period and achieved a better performance in the videogame, which is considered part of his daily life and a meaningful activity to him.
\end{abstract}

Keywords: Cognitive Dysfunction, Video Games, Rehabilitation, Technology. Autor para correspondência: Thiago da Silva Dias, Núcleo de Teoria e Pesquisa do Comportamento, Universidade Federal do Pará, Rua Augusto
Corrêa, 01, CEP 66075-110, Belém, PA, Brasil, e-mail: thiagodias.to@gmail.com

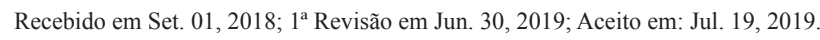

(1) Este é um artigo publicado em acesso aberto (Open Access) sob a licença Creative Commons Attribution, que permite uso, distribuição e reprodução em qualquer meio, sem restrições desde que o trabalho original seja corretamente citado. 


\section{Introdução}

Os avanços tecnológicos, continuamente, influenciam a prática dos profissionais de saúde e podem auxiliar na criação de novas ferramentas de intervenção. Desse modo, recentemente, a terapia ocupacional vem utilizando os princípios $\mathrm{da}$ realidade virtual (RV), que é definida como uma abordagem tridimensional gerada por computador que tem a capacidade de simular um ambiente real vivido pelo usuário através da estimulação dos canais sensoriais, por meio de hardwares e softwares específicos (RODRIGUES; BUSARELLO, 2017; SKJÆRET et al., 2016).

Neste sentido, o console de videogame Nintendo ${ }^{\circledR}$ Wii (NW) se enquadra na abordagem da RV e tem sido usado na reabilitaçáo de pacientes com desordens neurológicas, tais como a paralisia cerebral (PC), para a melhora de habilidades cognitivas e motoras (ANGUERA et al., 2013; DIAS et al., 2017; DE GRANDE; GALVÃO; GONDIM, 2011; MOREIRA, 2012). Estudos mostram que a interação do sujeito com este recurso permite a execução de movimentos utilizados no desempenho das atividades de vida diária, estimulando, assim, a funcionalidade cotidiana (MOREIRA, 2012; CAIANA; NOGUEIRA; LIMA, 2016).

Neste estudo, conforme descrito por Dias et al. (2017), a utilização de vídeo games como recursos terapêuticos integra o método de "gameterapia", o qual tem o potencial de viabilizar o processo de estimulação motora e/ou cognitiva. Especificamente, esse método pode viabilizar melhorias em habilidades perceptuais, atenção seletiva, concentração, pensamento abstrato, memória, organização viso-espacial, criatividade, funções executivas, entre outras habilidades cognitivas, por meio do aprendizado de habilidades e aquisição de conhecimento no contexto do jogo, o que facilita o aprendizado de novas tarefas em outros contextos (GREEN; BAVELIER, 2015; GRIFFITHS; KUSS; GORTARI, 2017).

No caso de sujeitos com PC, conforme Monteiro et al. (2011), Moreira (2012) e Moura et al. (2017), apesar de apresentarem primariamente desordens motoras, o comprometimento no cérebro pode acarretar alteraçóes sensoriais, comportamentais e cognitivas. Dessa forma, a PC pode abranger limitações em funçóes executivas, no processo de aprendizado e no desempenho de atividades cotidianas, visto que o quadro clínico pode causar restriçóes no processo de estimulação da capacidade de assimilação, retenção e elaboração do pensamento.
Neste contexto, considera-se importante a análise de atividade desempenhada pelo terapeuta ocupacional no âmbito do método de gameterapia, incluindo análise dos aspectos inerentes à jogabilidade, aos tipos de jogos, ao tempo e ao ambiente, bem como a análise de estratégias para promover o desenvolvimento de habilidades cognitivas a sujeitos com PC (MORAES et al., 2016).

Destaca-se que os estudos realizados junto a indivíduos com desordens neuromotoras, incluindo PC, abordam ganhos no desempenho ocupacional, função motora grossa, equilíbrio, força, nas atividades cotidianas, na função de membros superiores, na aderência ao tratamento e na motivação (DIAS et al., 2017; KASSEE et al., 2017; TARAKCI et al., 2016; UYSAL; BALTACI, 2016). Assim, verifica-se a predominância de estudos que relacionam o uso da gameterapia para estimulaçáo de aspectos motores. Nesse sentido, buscou-se ampliar esta perspectiva clínica, de modo a identificar os benefícios do videogame para as habilidades cognitivas de sujeitos com PC.

Desse modo, objetivou-se analisar as contribuições da gameterapia para as habilidades cognitivas de um adolescente com PC.

\section{Método}

Este estudo consiste em um estudo de caso, no qual o uso da gameterapia junto a um adolescente com PC foi analisado de forma detalhada.

\subsection{Aspectos éticos}

O projeto de pesquisa foi submetido à avaliação de um Comitê de Ética em Pesquisa (CEP) envolvendo seres humanos, do qual recebeu o Certificado de Apresentação para Apreciação Ética (CAAE) de no 30678214.2.0000.5174 e parecer de aprovação de no 673965. Consonantemente, o cuidador/responsável e o sujeito da pesquisa foram esclarecidos sobre os objetivos e implicaçóes do estudo, mediante a leitura e explicação de um Termo de Consentimento Livre e Esclarecido (TCLE) e de um Termo de Assentimento Livre e Esclarecido.

\subsection{Local da pesquisa}

A pesquisa foi conduzida no Núcleo de Desenvolvimento em Tecnologia Assistiva e Acessibilidade da Universidade do Estado do Pará (NEDETA/UEPA). Nesta instituição há uma sala projetada especificamente para o desenvolvimento de assistência e pesquisa utilizando o videogame 
como ferramenta terapêutica para a reabilitação de pacientes com sequelas neurológicas, incluindo PC.

\subsection{Sujeito da pesquisa}

O sujeito do estudo de caso é do sexo masculino e tem 12 anos. Apresenta PC do tipo Atetóide e mostra habilidades compatíveis com o nível I do Sistema de Classificação da Função Motora Grossa (GMFCS), visto que é independente na deambulação dentro e fora de casa e desempenha múltiplas atividades cotidianas sem utilizar ajudas técnicas para a mobilidade, o que o distingue de uma pessoa no nível II do GMFCS. Este sistema abrange cinco níveis e pode ser utilizado para classificar a função motora grossa de indivíduos com PC (PALISANO et al., 2007). Além disso, o participante mostra preferência por brincadeiras com bonecos, carros e aparelhos eletrônicos (celular e tablet), cursa o quarto ano do Ensino Fundamental em uma instituição de ensino regular, utiliza transporte coletivo urbano e frequenta praças, parques e shoppings no tempo livre. As informaçôes supracitadas foram coletadas a partir do instrumento de avaliação padrão do NEDETA/UEPA.

Com base em observação do desempenho durante a avaliação terapêutica ocupacional, verificou-se um padráo motor caracterizado por movimentos involuntários lentos, suaves e contorcidos de membros superiores, o qual acarreta dificuldades de coordenação motora global e fina, associadas à dissinergia de grupos musculares opostos, o que prejudica a realização de movimentos como flexão, extensão, pronação e supinação.

Adicionalmente, identificou-se que, apesar do déficit de equilíbrio dinâmico decorrente do padrão motor supracitado, o adolescente mostra deambulaçáo independente. Além disso, este apresenta comunicação por meio de linguagem não-verbal (gestos) e mostra independência nas interações sociais.

Para preservar a identidade do sujeito da pesquisa, seu nome foi substituído pelo pseudônimo Yoshi.

\subsection{Instrumentos e materiais de coleta de dados}

O Software Desenvolve ${ }^{\circledR}$ é um instrumento avaliativo que tem como principal referência a área cognitiva do Guia Portage Operacionalizado, de modo que possibilita a avaliaçáo diante de 19 habilidades cognitivas, distribuídas em 127 telas. Atualmente, na versão em tablet, o Desenvolve ${ }^{\circledR}$ é sensível para avaliar crianças com déficit na cognição e tem o potencial de atender às dificuldades motoras dos sujeitos com PC a partir do sistema de escaneamento ou varredura (OLIVEIRA; ASSIS; GAROTTI, 2014; OLIVEIRA; PINTO; RUFFEIL, 2004).

As habilidades avaliadas pelo software incluem: Percepçáo de Objetos do Cotidiano (POC); Percepção de Tamanho (PT); Percepção de Sequência (PES); Noção de Espaço (NE); Percepção Auditiva (PA); Identificando Açóes (IA); Percepção de Formas (PF); Esquema Corporal (EC); Associação de Iguais e Diferentes (AID); Percepção de Cores (PEC); Noção de Quantidade (NQ); Noção de Tempo (NT); Percepção de Letras e Números (PLN); Associação de Conjuntos (AC); Percepção Espaço-Temporal (PET); Noção de Sequência Numérica (NSN); Nomeação de Números (NN); Associação de Palavra ao Objeto (APO); e Identificação de Fatos pela Sequência de Açôes (IFSA).

O escore pode ser obtido por meio de porcentagem (\%), de modo a adquirir um percentual total - soma das dezenove habilidades - ou escores individuais (resultados de cada categoria).

Além disso, foi realizada avaliação contínua do desempenho da criança nos jogos por meio de anotaçôes nas folhas de registro padrão do setor de gameterapia do NEDETA/UEPA, a fim de coletar dados qualitativos relevantes observados ao longo das sessôes.

\subsection{Etapas do estudo}

O estudo foi dividido em cinco etapas: 1) Seleção do sujeito; 2) Avaliação pré-intervenção; 3) Elaboração do plano de intervençáo, incluindo análise e seleção do jogo; 4) Intervenções; e 5) Avaliação Pós-Intervenção.

\subsubsection{Seleção do sujeito}

Os critérios de inclusão foram: diagnóstico de PC; idade entre 10 e 18 anos; habilidades motoras compatíveis com os níveis I a III no GMFCS; capacidade de se comunicar e interagir com os pesquisadores; e assiduidade ao NEDETA

Os critérios de exclusão foram inadequação aos critérios supracitados, bem como deficiência intelectual, doenças cardiorrespiratórias e ocorrência de episódios convulsivos.

\subsubsection{Avaliação pré-intervenção}

O software Desenvolve ${ }^{\circledR}$ foi utilizado para a avaliação das habilidades cognitivas do sujeito pré-intervenção. O processo de avaliação requereu duas sessóes de, em média, 45 minutos, realizadas no intervalo de uma semana. 


\subsubsection{Elaboração do plano de intervenção}

Com base nos dados do Desenvolve ${ }^{\circledR}$, pôde-se traçar o plano de intervenção para o sujeito da pesquisa, com foco nas habilidades cognitivas com escores abaixo do percentil de $75 \%$. Esta etapa incluiu a análise e escolha do jogo mais adequado para atender às demandas identificadas.

\subsubsection{Análise e seleção do jogo}

O jogo Big Brain Academy: Wii Degree foi selecionado para a intervençáo, visto que este apresenta 15 minijogos divididos em cinco categorias ( 3 em cada) que contemplam algumas habilidades cognitivas avaliadas por meio do Desenvolve ${ }^{\circledR}$ : identificação lógica, memória, razão, matemática e comparação visual (CRAVEN; FABRICATORE, 2016).

Cada minijogo contém dez tentativas, e, ao final, é dado o percentual de acertos na partida, o tempo de execução do jogo e o valor da massa encefálica (de maneira simbólica) adquirida pelo participante.

Neste sentido, pôde-se favorecer o processo de aprendizagem ao sujeito com PC, selecionando os jogos mais adequados para os déficits identificados na avaliação. Na Tabela 1 foram elencados os jogos utilizados, as habilidades estimuladas e os procedimentos.

\subsubsection{Intervenções}

O jogo foi utilizado durante 10 sessóes, ocorridas em um período de 2 meses. Todas as intervençóes foram filmadas e fotografadas. A pontuação do sujeito no jogo foi registrada por meio de folha de registro, a qual contém onze sessôes, pois a primeira correspondeu a uma sessão-treino, de modo a ensinar o uso e identificar a usabilidade do videogame.

\subsubsection{Avaliação pós-intervenção}

Após as sessóes de gameterapia, foi realizada a reaplicação do Desenvolve ${ }^{\circledR}$ para avaliação das habilidades cognitivas do sujeito pós-intervenção.

\subsection{Análise dos resultados}

Os dados quanto às habilidades cognitivas foram analisados de forma quantitativa, de modo que foram estabelecidas comparaçóes, por meio de gráficos elaborados no software Excel, entre os escores das habilidades cognitivas pré e pós-intervenção. Além disso, os dados qualitativos coletados foram organizados em padróes e serão apresentados de forma discursiva.

\section{Resultados e Discussão}

\subsection{Avaliação pré-intervenção}

Com relação à avaliação inicial, os escores de Yoshi nas 19 habilidades cognitivas foram elencados na Figura 1. O sujeito obteve pontuação geral de 73,69\%, com 97 acertos e 30 erros, dentre as 127 questóes.

Neste contexto, considerando o percentil de $75 \%$, Yoshi obteve escores baixos em 10 habilidades: percepção de sequência (50\%), identificação de

Tabela 1. Minijogos utilizados no jogo Big Brain Academy: Wii Degree.

\begin{tabular}{|c|c|c|}
\hline Jogo & Habilidades & Procedimento \\
\hline Whack Match & $\begin{array}{l}\text {-Percepção de formas; } \\
\text {-Associação de iguais e diferentes; } \\
\text {-Atenção/concentração; }\end{array}$ & $\begin{array}{l}\text { Selecionar, em tempo hábil, as figuras na } \\
\text { parte inferior da tela, que mudam em curtos } \\
\text { intervalos de tempo, idênticas aos modelos } \\
\text { mostrados na parte superior. }\end{array}$ \\
\hline Face Case & $\begin{array}{l}\text {-Memória; } \\
\text {-Atenção/concentração; }\end{array}$ & $\begin{array}{l}\text { Selecionar dentre três opções a correspondente } \\
\text { a um rosto previamente mostrado na tela }\end{array}$ \\
\hline Balloon Burst & $\begin{array}{l}\text {-Noção de sequência numérica; } \\
\text {-Nomeação de números; } \\
\text {-Atenção/concentração; }\end{array}$ & $\begin{array}{l}\text { "Estourar" balões com numerais mostrados na } \\
\text { tela de acordo com a ordem crescente. }\end{array}$ \\
\hline Mallet Match & $\begin{array}{l}\text {-Noção de quantidade; } \\
\text {-Percepção de letras e números; } \\
\text {-Atenção/concentração; }\end{array}$ & $\begin{array}{l}\text { Eliminar um ou mais cubos com numerais de } \\
\text { uma coluna mostrada na parte inferior da tela } \\
\text { para que a soma corresponda a um numeral } \\
\text { mostrado na parte superior. }\end{array}$ \\
\hline Species Spotlight & $\begin{array}{l}\text {-Percepção de formas; } \\
\text {-Associação de iguais e diferentes; } \\
\text {-Memória; } \\
\text {-Atenção/concentração; } \\
\text {-Associação de conjuntos; } \\
\text {-Noção de quantidade; }\end{array}$ & $\begin{array}{l}\text { Identificar o conjunto de animais que está em } \\
\text { maior quantidade dentre três opções mostradas } \\
\text { na tela. }\end{array}$ \\
\hline
\end{tabular}


ações (67\%), associação de iguais e diferentes (0\%), noção de quantidade (50\%), noção de tempo (43\%), percepção de letras e números (68\%), associação de conjuntos (50\%), percepção espaço-temporal (67\%), nomeação de números (67\%), identificação de fatos pela sequência de açóes (67\%).

Os resultados obtidos neste estudo foram comparados a escores verificados em outras pesquisas que utilizaram o software Desenvolve ${ }^{\circledR}$ como instrumento de avaliaçáo para sujeitos com PC (OLIVEIRA; PINTO; RUFFEIL, 2004) e síndrome de Down (PINHEIRO et al., 2012), nas quais foi identificada uma média de $35,6 \%$ de erros por parte dos participantes, bem como dificuldades, principalmente, nas seguintes categorias: noção de quantidade, associação de iguais e diferentes, noção de sequência numérica e nomeação de números.

\subsection{Avaliação pós-intervenção}

Após a realização das 10 intervenções com gameterapia, foi feito um comparativo com o percentual obtido antes e após a intervenção por meio do Software Desenvolve ${ }^{\circledR}$ (Figura 2). Após a intervenção, a pontuação geral de Yoshi aumentou para 90,89\%, com 112 acertos e 15 erros.

Destaca-se que houve aumento na porcentagem de acertos em cinco habilidades cognitivas diretamente

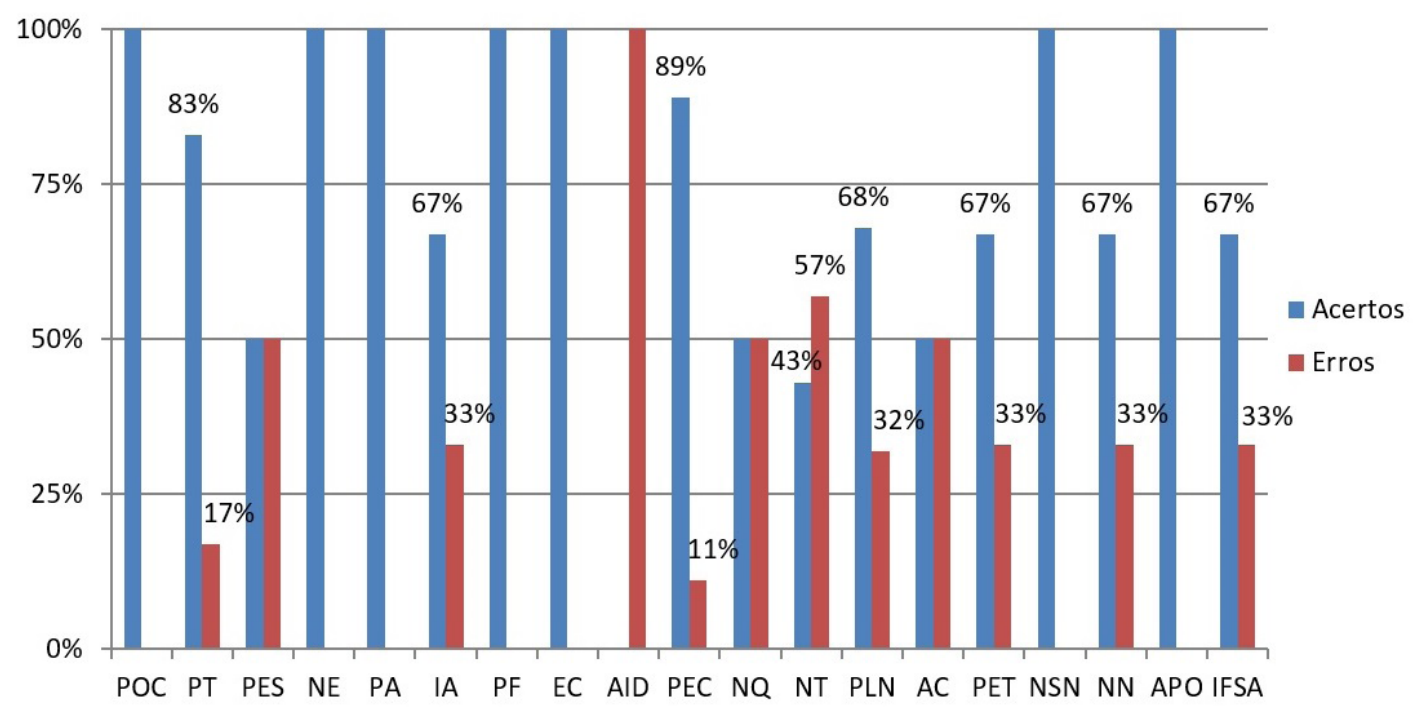

Figura 1. Escores do sujeito no software Desenvolve ${ }^{\circledR}$ pré-intervenção. Fonte: Pesquisa de campo.

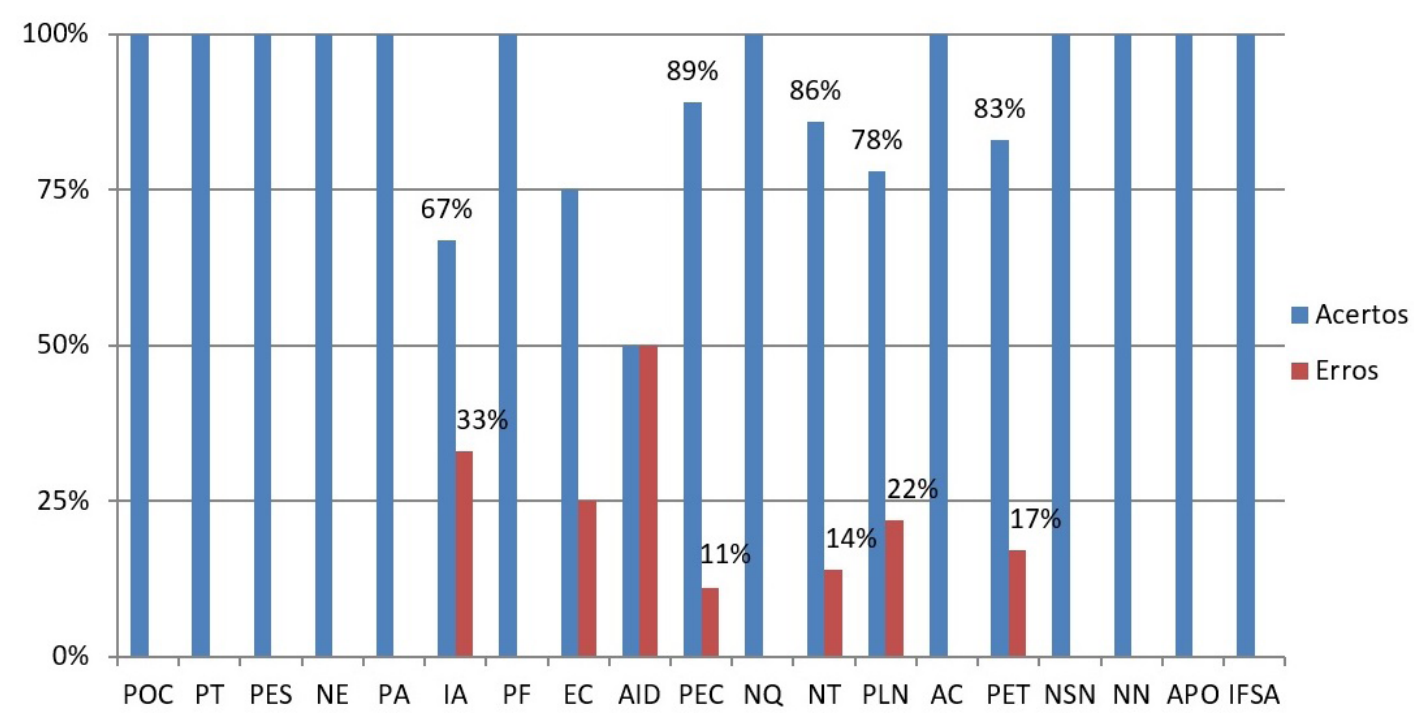

Figura 2. Escores do sujeito no software Desenvolve ${ }^{\circledR}$ pós-intervenção. Fonte: Pesquisa de campo. 
estimuladas pelo recurso: AID - associação de iguais e diferentes (de $0 \%$ para $50 \%$ ), AC - associação de conjuntos (de 50\% para 100\%), NQ - noção de quantidade (de $50 \%$ para $100 \%$ ), NN - nomeação de números (de 67\% para 100\%) e PLN - percepção de letras e números (de 68\% para 78\%).

Além disso, observou-se que outras cinco habilidades também obtiveram maior percentual apesar de não serem diretamente estimuladas pelos jogos do videogame: NT -noçáo de tempo (de $43 \%$ para $86 \%$ ), IFSA - identificação de fatos pela sequência de açóes (de 67\% para 100\%), PT - percepção de tamanho (de $83 \%$ para $100 \%$ ), PES - percepção de sequência (de 50 para 100\%) e PET - percepção espaço-temporal (de 67\% para $83 \%$ ).

\subsection{Interpretação}

Os resultados deste estudo sugerem que a intervenção utilizando o videogame pode ter contribuído para a melhora de dez habilidades cognitivas, sendo cinco estimuladas diretamente por meio dos jogos selecionados. Quanto às outras nove habilidades avaliadas pelo Desenvolve ${ }^{\circledR}$, foi identificada manutenção das habilidades, visto que houve uma estabilidade na porcentagem de acertos de, em média, 95,10\%.

Ressalta-se que o sujeito deste estudo de caso obteve escore inferior a $75 \%$ em nove habilidades que coincidem com as habilidades deficitárias encontradas nos estudos de Oliveira, Pinto e Ruffeil (2004) e Pinheiro et al. (2012), nos quais o Desenvolve ${ }^{\circledR}$ foi utilizado para avaliar sujeitos com deficiência.

Neste sentido, destaca-se que as características do quadro clínico e a pouca participação do sujeito com PC no processo de reabilitação cognitiva, incluindo escassez de estímulos sensoriais, educacionais e sociais, pode causar um atraso ou déficit na aquisição de habilidades cognitivas. Assim, o conhecimento que deveria ter sido adquirido em sua faixa etária pode ser prejudicado pela limitação motora (OLIVEIRA, 2010; FREIRE, 2016; HOFFMANN; TAFNER; FISCHER, 2003). Entretanto, por meio da estimulação da neuroplasticidade, os sujeitos com PC podem apresentar melhoras em habilidades motoras e cognitivas (OLIVEIRA; PINTO; RUFFEIL, 2004; MORGAN et al., 2016).

Sobre a temática, Dederich (2000) afirma que existem dois fatores capazes de influenciar as habilidades cognitivas: a exploração do meio e os estímulos advindos desse meio. Consonantemente, Mulder et al. (2017) indicam que no processo de desenvolvimento cognitivo, a criança está exposta à influência contínua e recíproca de fatores internos e externos (ambientais). Da mesma forma, Tabaquim, Ribeiro e Ciasca (2016) destacam que a lesão pode ocasionar limitaçóes na participação do sujeito, o que influencia o amadurecimento do Sistema Nervoso Central (SNC) e, consequentemente, na aprendizagem de funçôes executivas.

Desse modo, os indivíduos com PC podem apresentar prejuízos na cognição em virtude dos déficits motores que limitam as experiências sensoriais e perceptivas e, assim, ocorre atraso no desenvolvimento. Em muitos casos, a capacidade cognitiva do sujeito não está comprometida, necessitando somente ser inserido em programas de reabilitaçáo adequados (OLIVEIRA; PINTO; RUFFEIL, 2004).

Nesta perspectiva, ressalta-se que Yoshi não apresentava dificuldades severas para apreender novos conceitos, entretanto apresentava maior lentificação para executar a tarefa devido ao seu quadro motor.

Sobre a temática, Miller e Clark (2002) destacam que uma característica de pessoas com disfunção neuromotora do tipo atetóide é a possível preservação das habilidades cognitivas, bem como, esses sujeitos podem adquirir linguagem compreensiva, apesar dos déficits na produção de fala.

Complementarmente às melhoras cognitivas, os dados coletados por meio das folhas de registro ao longo das sessóes de gameterapia evidenciaram aumento na motivação para a realização das atividades e na assiduidade ao tratamento. Neste sentido, destaca-se que o aspecto lúdico, divertido e desafiador do jogo estimula o sujeito a desenvolver a sua criatividade e imaginação, concomitante ao desenvolvimento de habilidades específicas por meio da tomada de decisóes e desenvolvimento de estratégias para progressão de níveis (ALVES, 2008).

Neste contexto, o sujeito constrói e orienta seu processo de desenvolvimento cognitivo através da motivação, da atenção e da ação. O indivíduo com $\mathrm{PC}$, geralmente, não tem limitações relacionadas à motivação, curiosidade, senso de humor, atenção e/ou sentimento de prazer durante atividades lúdicas, porém suas dificuldades encontram-se no plano da execução motora, de modo que podem apresentar limitaçóes em diversas atividades cotidianas decorrentes de barreiras no acesso a brinquedos, dificuldades de manuseio, condições ambientais insatisfatórias, déficits em habilidades interpessoais, entre outros (BRAGA; GRACIANI, 2015; DEDERICH, 2000).

Em contrapartida, com a utilização do NW, a própria jogabilidade e os recursos do videogame foram capazes de se adequar à demanda física de 
Yoshi e, desse modo, foi possível favorecer a melhora de habilidades cognitivas.

O feedback imediato viabilizado pelas dez tentativas (repetiçôes) de cada minijogo do Big Brain Academy: Wii Degree é um elemento central do processo terapêutico, visto que, conforme demonstrado por Schiavinato et al. (2010) e Zoccolillo et al. (2015), as múltiplas tentativas em jogos de videogame podem estimular o cérebro e cerebelo para a correçâo dos erros durante a repetição das tarefas funcionais inerentes aos jogos, promovendo estímulos com variabilidade sensorial (visual, auditivo e, eventualmente, táteis), estimulando a motivação do jogador e, consequentemente, viabilizando oportunidades para o aprimoramento do processo de aprendizagem, incluindo feedback cognitivo por meio de escores e consequência das açôes no jogo.

Tabaquim (1996, p. 32) destaca que

[...] o sujeito que apresenta PC torna-se passivo durante as atividades, perdendo a oportunidade de realizar os próprios ajustamentos, ajustamentos estes que contribuem na aprendizagem.

Neste contexto, Zampieri, Santos e Pfeifer (2016) reiteram que a capacidade de interpretar as informaçóes ambientais (sensoriais e cognitivas) podem ocorrer em consequência das limitaçôes de atividade que restringem o aprendizado, a exploração do ambiente, a movimentação ativa e a aquisição de um repertório sensorial, perceptual e cognitivo.

Portanto, faz-se necessária a repetição das atividades e açôes, o que é efetivado pela gameterapia, promovendo ao indivíduo a oportunidade de aprender por tentativas e erros, com adequada exploração do meio.

\section{Conclusão}

A introdução de recursos tecnológicos nos processos terapêuticos ocupacionais se apresenta como uma estratégia para atender às demandas dos sujeitos que exploram e acompanham as inovaçóes tecnológicas, incluindo sujeitos que apresentam alguma deficiência neuromotora. Desse modo, a intervençáo no escopo da terapia ocupacional visa a melhora do desempenho em uma atividade significativa (jogar videogame) para o sujeito e, concomitantemente, a melhora de habilidades cognitivas subjacentes a outras atividades cotidianas.

Desse modo, as sessóes de gameterapia podem ter contribuído para a aquisiçáo e o aprimoramento de habilidades cognitivas pelo sujeito da pesquisa, levando em consideração a incorporação de um recurso que estimule a motivação e a participação no processo de terapêutico, visto que esta é considerada uma atividade de lazer para o sujeito. Portanto, a utilizaçáo de uma abordagem diferenciada da terapia convencional pode ter permitido ao indivíduo a atribuiçáo de um novo sentido ao seu processo terapêutico.

Verificou-se assim, através da pesquisa, que a gameterapia pode se apresentar como estratégia terapêutica ocupacional, por possibilitar melhora nas habilidades cognitivas e no engajamento do paciente no tratamento, sendo aliada a outros métodos de tratamento de indivíduos com PC.

Nesta perspectiva, ressalta-se a importância da realização de estudos na área de gameterapia com enfoque em habilidades cognitivas, visto que a proporção de estudos com enfoque nestas habilidades é muito menor quando comparada a estudos que investigam a utilização de videogames para a estimulação motora, embora, conforme mostrado neste estudo, este recurso possa contribuir para a viabilização da aquisição e/ou aprimoramento de habilidades cognitivas.

Ressalta-se que, nesta pesquisa, o uso do método de estudo de caso configura uma limitação quanto à validade externa e ao controle de variáveis, o que limita a generalização dos resultados. Portanto, sugere-se a utilização de grupos-controle em pesquisas futuras.

\section{Referências}

ALVES, L. Análise das performances em teste de atenção sustentada: comparação entre jogadores e não-jogadores de videogame. 2008. 102 f. Dissertação (Mestrado em Ciências da Saúde) - Universidade Federal de Minas Gerais, Belo Horizonte, 2008. Disponível em: <http://www. bibliotecadigital.ufmg.br/dspace/bitstream/handle/1843/ ECJS-7KUGCY/luciana_alves_disserta_o.pdf?sequence $=1$ > Acesso em: 31 ago. 2018.

ANGUERA, J. A. et al. Video game training enhances cognitive control in older adults. Nature, Inglaterra, v. 501, n. 7465, p. 97-101, 2013. Disponível em: <https:// www.nature.com/articles/nature12486>. Acesso em: 31 ago. 2018.

BRAGA, M. A. M.; GRACIANI, Z. O brincar na rotina da criança com paralisia cerebral. Cadernos de Pós-graduação em Distúrbios do Desenvolvimento, São Paulo, v. 15, n. 1, p. 41-49, 2015. Disponível em: <http://editorarevistas. mackenzie.br/index.php/cpgdd/article/view/11268>. Acesso em: 31 ago. 2018.

CAIANA, T. L.; NOGUEIRA, D. L.; LIMA, A. C. D. A realidade virtual e seu uso como recurso terapêutico ocupacional: revisão integrativa. Cadernos de Terapia Ocupacional da UFSCar, São Carlos, v. 24, n. 3, p. 575-589, 2016. 
Disponível em: <http://www.cadernosdeterapiaocupacional. ufscar.br/index.php/cadernos/article/view/1218>. Acesso em: 31 ago. 2018.

CRAVEN, M. P.; FABRICATORE, C. Game features of cognitive training. In: INTERNATIONAL CONFERENCE ON INTERACTIVE TECHNOLOGIES AND GAMES, 9., 2016, Nottingham. Anais... Nottingham: Institute of Electrical and Electronics Engineers, Inc., 2016. p. 42-49. Disponível em: <https://ieeexplore.ieee.org/abstract/document/ 7782513/>. Acesso em: 31 ago. 2018.

DE GRANDE, A. A. B.; GALVÃO, F. R. O.; GONDIM, L. C. A. Reabilitação virtual através do videogame: relato de caso no tratamento de um paciente com lesão alta dos nervos mediano e ulnar. Acta Fisiátrica, São Paulo, v. 18, n. 3, p. 157-162, 2011. Disponível em: <http://www. revistas.usp.br/actafisiatrica/article/view/103644>. Acesso em: 31 ago. 2018.

DEDERICH, A. C. Desenvolvimento cognitivo e linguagem na paralisia cerebral. Cefac, 2000. Disponível em: <http://www.cefac.br/library/teses/ 39b1aec2b9bf05903941e267aae39d8f.pdf>. Acesso em: 31 ago. 2018.

DIAS, T. S. et al. As contribuiçôes da gameterapia no desempenho motor de indivíduo com paralisia cerebral. Cadernos de Terapia Ocupacional da UFSCar, São Carlos, v. 25, n. 3, p. 575-584, 2017. Disponível em: <http:// www.cadernosdeterapiaocupacional.ufscar.br/index.php/ cadernos/article/view/1667>. Acesso em: 31 ago. 2018.

FREIRE, T. C. Associação entre funçóes executivas e o desenho na idade pré-escolar: comparaçóes entre paralisia cerebral e desenvolvimento típico. 2016. 86 f. Dissertação (Mestrado em Distúrbio do Desenvolvimento) - Universidade Presbiteriana Mackenzie, São Paulo, 2016. Disponível em: <http://tede.mackenzie.br/jspui/handle/tede/2968>. Acesso em: 31 ago. 2018.

GREEN, C. S.; BAVELIER, D. Action video game training for cognitive enhancement. Current Opinion in Behavioral Sciences, Reino Unido, v. 4, p. 103-108, 2015. Disponível em: <https://www.sciencedirect.com/science/article/pii/ S2352154615000613>. Acesso em: 31 ago. 2018.

GRIFFITHS, M. D.; KUSS, D. J.; GORTARI, A. B. O. Videogames as therapy: an updated selective review of the medical and psychological literature. International Journal of Privacy and Health Information Management, Estados Unidos, v. 5, n. 2, p. 71-96, 2017. Disponível em: <https:/www.igi-global.com/article/videogames-astherapy/182880>. Acesso em: 31 ago. 2018.

HOFFMANN, R. A.; TAFNER, M. A.; FISCHER, J. Paralisia cerebral e aprendizagem: um estudo de caso inserido no ensino regular. Revista Leonardo Pós, Blumenau, v. 2, p. 1-15, 2003. Disponível em: <http://atividadeparaeducacaoespecial.com/wpcontent/ uploads/2014/07/PC-E-APRENDIZAGEM.pdf $>$. Acesso em: 31 ago. 2018.

KASSEE, C. et al. Home-based Nintendo Wii training to improve upper-limb function in children ages 7 to 12 with spastic hemiplegic cerebral palsy. Journal of Pediatric Rehabilitation Medicine, Holanda, v. 10, p. 145-154, 2017. Disponível em: <https://content.iospress.com/articles/ journal-of-pediatric-rehabilitation-medicine/prm439>. Acesso em: 31 ago. 2018.

MILlER, G.; CLARK, G. F. Paralisias cerebrais. São Paulo: Manole, 2002.

MONTEIRO, C. B. M. et al. Paralisia cerebral e aprendizagem de jogo eletrônico (Nintendo Wii). In: MONTEIRO, C. B. M. (Org.). Realidade virtual na paralisia cerebral. São Paulo: Plêiade, 2011. p. 111-142.

MORAES, V. B. et al. O uso do videogame Nintendo Wii como recurso terapêutico para idosos: uma análise da atividade na perspectiva da terapia ocupacional. Cadernos de Terapia Ocupacional da UFSCar, São Carlos, v. 24, n. 4, p. 705-714, 2016. Disponível em: <http:// www.cadernosdeterapiaocupacional.ufscar.br/index.php/ cadernos/article/view/1383 >. Acesso em: 31 ago. 2018.

MOREIRA, M. C. A utilização da realidade virtual como intervenção terapêutica para a melhora do controle postural e da mobilidade funcional em crianças com paralisia cerebral. 2012. 128 f. Dissertação (Mestrado em Fisioterapia) - Universidade Federal de Pernambuco, Recife, 2012. Disponível em: <https://repositorio.ufpe.br/handle/123456789/10836>. Acesso em: 31 ago. 2018.

MORGAN, C. et al. Single blind randomised controlled trial of GAME (goals - activity - motor enrichment) in infants at high risk of cerebral palsy. Research in Developmental Disabilities, Reino Unido, v. 55, p. $256-$ 267, 2016. Disponível em: <https://www.sciencedirect. com/science/article/pii/S0891422216300750>. Acesso em: 31 ago. 2018.

MOURA, R. et al. Mini-mental state exam for children (MMC) in children with hemiplegic cerebral palsy. Dementia \& Neuropsychologia, São Paulo, v. 11, n. 3, p. 287-296, 2017. Disponível em: <http://www.scielo.br/ $\mathrm{pdf} / \mathrm{dn} / \mathrm{v} 11 \mathrm{n} 3 / 1980-5764-\mathrm{dn}-11-03-0287 . \mathrm{pdf}>$. Acesso em: 31 ago. 2018.

MULDER, H. et al. How children learn to discover their environment: an embodied dynamic systems perspective on the development of spatial cognition. In: POSTMA, A.; VAN DER HAM, J. M. Neuropsychology of space: spatial functions of the human brain. Holanda: Elsevier Inc., 2017. p. 309-360.

OLIVEIRA, A. I. A.; ASSIS, G. J. A.; GAROTTI, M. F. Tecnologias no ensino de crianças com paralisia cerebral. Revista Brasileira de Educação Especial, Marília, v. 20, n. 1, p. 85-102, 2014. Disponível em: <http://www.scielo.br/ pdf/rbee/v20n1/a07v20n1.pdf>. Acesso em: 31 ago. 2018.

OLIVEIRA, A. I.; PINTO, R. F.; RUFFEIL, E. A tecnologia e o desenvolvimento cognitivo da criança com paralisia cerebral. Évora: Profala, 2004. Disponível em: <http:// www.profala.com/artdef3.pdf>. Acesso em: 31 ago. 2018.

OLIVEIRA, A. I. A. Integrando tecnologias para leitura em crianças com paralisia cerebral na educação inclusiva. 
2010. 145 f. Tese (Doutorado em Teoria e Pesquisa do Comportamento) - Universidade Federal do Pará, Belém, 2010. Disponível em: <http://ppgtpc.propesp.ufpa.br/ ARQUIVOS/teses/Ana\%20Irene\%202010.pdf>. Acesso em: 31 ago. 2018.

PALISANO, R. et al. GMFCS - E \& R: gross motor function classification system expanded and revised. Canadá: CanChild Centre for Childhood Disability Research, 2007. Disponível em: <https://www.canchild. $\mathrm{ca} /$ system/tenon/assets/attachments/000/000/075/original/ GMFCS-ER_Translation-Portuguese2.pdf>. Acesso em: 31 ago. 2018.

PINHEIRO, M. A. et al. Software desenvolve ${ }^{\circledR}$ e histórias infantis: contribuiçóes ao desenvolvimento cognitivo de crianças com síndrome de down. Informática na Educação: Teoria e Prática, Porto Alegre, v. 15, n. 2, p. 27-43, 2012. Disponível em: <http://www.seer.ufrgs.br/ InfEducTeoriaPratica/article/view/23177>. Acesso em: 31 ago. 2018.

RODRIGUES, T. N.; BUSARELLO, R. I. Realidade virtual e gamificação: um estudo sobre realidades imersivas no processo de aprendizagem. Revista Estética, São Paulo, v. 11, n. 11, p. 1-7, 2017. Disponível em: <http://www. usp.br/estetica/index.php/estetica/article/view/92>. Acesso em: 31 ago. 2018.

SCHIAVINATO, A. M. et al. Influência da realidade virtual no equilíbrio de paciente portador de disfunção cerebelar - estudo de caso. Revista Neurociências, São Paulo, v. 19, n. 1, p. 119-127, 2010. Disponível em: <https:// www.unip.br/presencial/comunicacao/publicacoes/ics/ edicoes/2010/01_jan-mar/V28_n1_2010_p50-52.pdf>. Acesso em: 31 ago. 2018.

SKJÆRET, N. et al. Exercise and rehabilitation delivered through exergames in older adults: an integrative review of technologies, safety and efficacy. International Journal of Medical Informatics, Irlanda, v. 85, n. 1, p. 1-16, 2016.
Disponível em: <https://www.sciencedirect.com/science/ article/pii/S1386505615300514>. Acesso em: 31 ago. 2018.

TABAQUIM, M. L. M. Paralisia cerebral: ensino de leitura e escrita. Bauru: EDUSC, 1996.

TABAQUIM, M. L. M.; RIBEIRO, M. V. L. M.; CIASCA, S. M. Aprendizagem e paralisia cerebral. In: ROTTA, N. T; OHLWEILER, L.; RIESGO, R. S. (Org.). Transtornos da aprendizagem: abordagem neurobiológica e multidisciplinar. Porto Alegre: Artmed, 2016. p. 427-433.

TARAKCI, D. et al. Effects of Nintendo Wii-Fit video games on balance in children with mild cerebral palsy. Pediatrics International, Japão, v. 58, n. 10, p. 1042-1050, 2016. Disponível em: <https://onlinelibrary.wiley.com/ doi/abs/10.1111/ped.12942>. Acesso em: 31 ago. 2018.

UYSAL, S. A.; BALTACI, G. Effects of Nintendo Wii ${ }^{\text {TM }}$ training on occupational performance, balance, and daily living activities in children with spastic hemiplegic cerebral palsy: a single-blind and randomized trial. Games for Health Journal, Estados Unidos, v. 5, n. 5, p. 311-317, 2016. Disponível em: <https://www.liebertpub.com/ doi/10.1089/g4h.2015.0102>. Acesso em: 31 ago. 2018.

ZAMPIERI, L. M.; SANTOS, J. L.; PFEIFER, L. I. Validade discriminante do protocolo do desempenho funcional e social de crianças com paralisia cerebral. Acta Fisiátrica, São Paulo, v. 23, n. 2, p. 66-72, 2016. Disponível em: <http://www.periodicos.usp.br/actafisiatrica/article/ view/137616>. Acesso em: 31 ago. 2018.

ZOCCOLILLO, L. et al. Video-game based therapy performed by children with cerebral palsy: a cross-over randomized controlled trial and a cross-sectional quantitative measure of physical activity. European Journal of Physical and Rehabilitation Medicine, Italy, v. 51, n. 6, p. 669-676, 2015. Disponível em: <https://europepmc.org/abstract/ med/25653079>. Acesso em: 31 ago. 2018.

\section{Contribuição dos Autores}

Os autores Thiago da Silva Dias e Karoline Faro da Conceição foram responsáveis pela concepção pesquisa bibliográfica coleta de dados análise interpretação e discussão dos resultados assim como redação final do artigo. Os autores Rafael Luiz Morais da Silva e Ana Irene Alves de Oliveira realizaram orientação teórica e metodológica do estudo e auxiliaram na análise e interpretação dos resultados. Todos os autores aprovaram a versão final do texto.

\section{Fonte de Financiamento}

Conselho Nacional de Desenvolvimento Científico e Tecnológico (CNPq).

\section{Notas}

${ }^{1}$ A pesquisa foi submetida à avaliação do Comitê de Ética em Pesquisa (CEP) envolvendo seres humanos, do qual recebeu o Certificado de Apresentação para Apreciação Ética (CAAE) de no 30678214.2.0000.5174 e parecer de aprovação de no 673965 , de modo que está de acordo com os procedimentos éticos vigentes. 